\title{
High-resolution investigation of metal nanoparticle growth on an insulating surface
}

\author{
Jeffrey M. Mativetsky, ${ }^{*}$ Shawn Fostner, Sarah A. Burke, ${ }^{\dagger}$ and Peter Grutter \\ Department of Physics, McGill University, Montreal, Canada H3A 2T8 \\ (Received 22 April 2009; revised manuscript received 27 June 2009; published 31 July 2009)
}

\begin{abstract}
The three-dimensional nanoparticle morphology and the nanoparticle-substrate relationship during the submonolayer growth of three metals (gold, tantalum, and palladium) on the alkali halide $\mathrm{KBr}(001)$ surface is investigated by combining in situ high-resolution noncontact atomic force microscopy and ex situ transmission electron microscopy approaches. Highly varied growth behavior between the metals is revealed. Gold produces nearly spherical multiply twinned nanoparticles at room temperature and an increasing number of epitaxial particles at elevated temperatures. In contrast, the tantalum grows as relatively flat fractal particles, despite the square symmetry of the substrate lattice, a condition which normally precludes fractal growth. The tantalum also exhibits a strong affinity for $\mathrm{KBr}$ surface steps, leading to one-dimensional chains of nanoparticles. The deposition of palladium results in the creation of protruding substrate distortions and monolayer-high rectangular $\mathrm{KBr}$ islands in addition to the growth of palladium nanoparticles. It is hypothesized that the unusual growth observed in the palladium- $\mathrm{KBr}$ system is caused by the interdiffusion of palladium under the $\mathrm{KBr}$ surface. The range of growth behavior in the three systems is described in terms of the surface and interface energies, yielding bounds on the metal/KBr interface energies.
\end{abstract}

DOI: 10.1103/PhysRevB.80.045430 PACS number(s): 68.55.A-, 61.46.Df, 68.37.Ps, 68.37.Lp

\section{INTRODUCTION}

Metal nanoparticles are finding use in a great range of applications from chemical sensors ${ }^{1}$ to prospective cancer therapies. ${ }^{2}$ This is largely because the size of the particles, between atomic scale and bulk, brings about new properties. For example, gold which is famous for its inert nature becomes reactive with various gases and a potential catalyst, when its particle dimensions are sufficiently small.,

Metal nanoparticles supported on insulating substrates are especially important for many applications including catalysis, where such systems are already ubiquitous. ${ }^{5}$ In fundamental studies, the nucleation and growth of particles during metal evaporation on alkali halide surfaces has provided a testbed for assessing models of thin-film growth. ${ }^{6}$ More recently, metal nanoparticles on surfaces are finding increasing use in nanofabrication, including as catalysts for carbon nanotube growth, ${ }^{7,8}$ catalysts for the vapor-solid-liquid growth of inorganic nanowires, ${ }^{9,10}$ and for nanoelectronics applications. ${ }^{11-13}$

Despite the importance of metal nanoparticle on insulator systems, the structural characterization of such samples presents a considerable experimental challenge: the nanoscopic dimensions of the nanoparticles require high-resolution microscopies while the insulating substrate limits the possibility of using electron-based or ion-based probes which may induce surface damage or charging effects. The first experimental studies of metal nanoparticles on insulators, in the 1950s, employed carbon replicas of metal decoration patterns as a means to learn about the surface structure of the underlying insulating substrate, which was inaccessible to high-resolution characterization at the time. ${ }^{14}$ Later electron microscopy studies on carbon replicas showed a rich "zoo" of nanoparticle shapes which can arise, including epitaxial particles (EPs) and various forms of multiply twinned nanoparticles (MTPs). ${ }^{15}$ More recently, atomic force microscopy (AFM) and, in particular, noncontact atomic force micros- copy (NC-AFM) has opened the possibility of directly probing the high-resolution structure of nanoparticles as well as the insulating host surface in situ, without the need for replicas. Under the controlled environment of ultrahigh vacuum, $\mathrm{NC}-\mathrm{AFM}$ has been shown to rival the resolution capabilities of scanning tunneling microscopy, which is limited to conducting substrates, demonstrating atomic resolution on insulators, ${ }^{16-19}$ semiconductors, ${ }^{20-22}$ metals, ${ }^{23,24}$ as well as molecular resolution on adsorbed molecular layers. ${ }^{25-27}$

To date, NC-AFM has only been applied to a handful of nanoparticle on insulator systems including gold on $\mathrm{KBr}$ (001), ${ }^{28-32}$ gold on $\mathrm{RbI}(001),{ }^{31}$ and palladium on $\mathrm{MgO}$ $(001) .{ }^{33}$ Earlier work on the gold/KBr system has revealed a "fuzzy" "hemispherelike" appearance of vacuum-deposited gold nanoparticles, which has been attributed to charging of the clusters. ${ }^{28}$ This has been supported by Kelvin probe measurements which exhibit the presence of additional electrostatic forces over the clusters ${ }^{30}$ and measurements of nucleation densities which show that the clusters are predominantly nucleated at charged defect sites. ${ }^{32}$

Here, we present the growth of three metals (gold, tantalum, and palladium) which exhibit drastically different behaviors from one another on an alkali halide surface, $\mathrm{KBr}$ (001). The range of growth morphologies from epitaxial nanocrystals to linear chains of fractal nanoparticles and growth which induces substrate surface modifications highlights the richness and complexity of the topic, which was previously largely inaccessible. In situ noncontact-AFM and ex situ transmission electron microscopy (TEM) were performed on the same samples in order to gain complementary insight into the nanoparticle morphology and the relationship between the adsorbate and the substrate.

\section{EXPERIMENTAL METHODS}

$\mathrm{KBr}$ (001) surfaces were prepared by cleaving a single crystal (supplied by Korth Krystalle, Germany) in ultrahigh 
vacuum (base pressure $<4 \times 10^{-8} \mathrm{~Pa}$ ). Following exposure of the clean (001) face, the samples were heated for $1 \mathrm{~h}$ at $150{ }^{\circ} \mathrm{C}$ to remove any residual surface charge. An Oxford Applied Research EGN4 electron-beam evaporator was used to deposit the metals onto the $\mathrm{KBr}$ surface. The evaporator is fitted with a charge-deviating grid, which is held at $2 \mathrm{kV}$. This reduces the amount of charge reaching the surface and hence decreases the number of charge-induced nucleation sites. $^{32,34}$ The total (positive $+\mid$ negative $\mid$ ) current-density incident on the sample during evaporation, measured according to procedure used in Ref. 34 was 220, 680, and $250 \mathrm{pA} / \mathrm{cm}^{2}$ during the deposition of gold, tantalum, and palladium, respectively. The beam of charge was estimated to consist of 60-70 \% positive ions (ionized background gas atoms and ionized evaporant atoms) and 30-40\% electrons. The ion gauges were kept off for the duration of the experiment since the charge given off by the gauges can lead to additional surface defects. The metal coverages and rates were measured with an Inficon quartz-crystal monitor. All coverages are quoted in terms of monolayers (MLs). A single ML is defined here as the number density of atoms required to cover each lattice site on the substrate with the $\mathrm{K}+\mathrm{Br}$ basis taken as a single lattice site. A rate of $0.025 \mathrm{ML} / \mathrm{min}$ was used for all samples.

NC-AFM characterization was performed in ultrahigh vacuum at room temperature using a JEOL JSPM 4500A AFM with a Nanosurf phase-locked loop and amplitude controller. The noncontact mode involves the use of the cantilever frequency for regulating the tip-sample separation. ${ }^{35,36}$ Cantilevers supplied by Nanosensors with a typical resonance frequency of $170 \mathrm{KHz}$ and spring constant of 40-50 $\mathrm{N} / \mathrm{m}$ were used with an oscillation amplitude of $5 \mathrm{~nm}$. Tips with a guaranteed radius of less than $10 \mathrm{~nm}$ (Nanosensors, PPP-NCLR) were employed. Measurements were only performed with tips which exhibited minimal and symmetric tip dilation effects. Prior to imaging, frequency shift versus voltage curves were acquired in order to evaluate the contact potential difference (CPD) between tip and sample. To reduce the electrostatic forces during imaging, a voltage equal to the CPD was applied.

The TEM measurements were performed on carboncoated replicas. These were prepared by depositing 8-10 nm of carbon onto the samples in a separate vacuum chamber and then floating the carbon film, with the embedded metal nanoparticles, in water onto a TEM grid. The TEM measurements were performed using a JEOL 2000FX and a JEOL JEM-2010 with an acceleration voltage of 80 and $200 \mathrm{kV}$, respectively.

\section{RESULTS AND DISCUSSION}

\section{A. Gold on $\mathrm{KBr}$ (001)}

Some general characteristics of the gold on $\mathrm{KBr}(001)$ growth at room temperature can be seen in Fig. 1. First, the island growth mode is followed with some particles forming on terraces and others decorating the steps. Much like in previous studies, ${ }^{28-32}$ the gold nanoparticles have a round, hemispherical appearance in the NC-AFM images [Figs. 1(a)-1(c)]. NC-AFM [Fig. 1(a)] also provides direct evi-
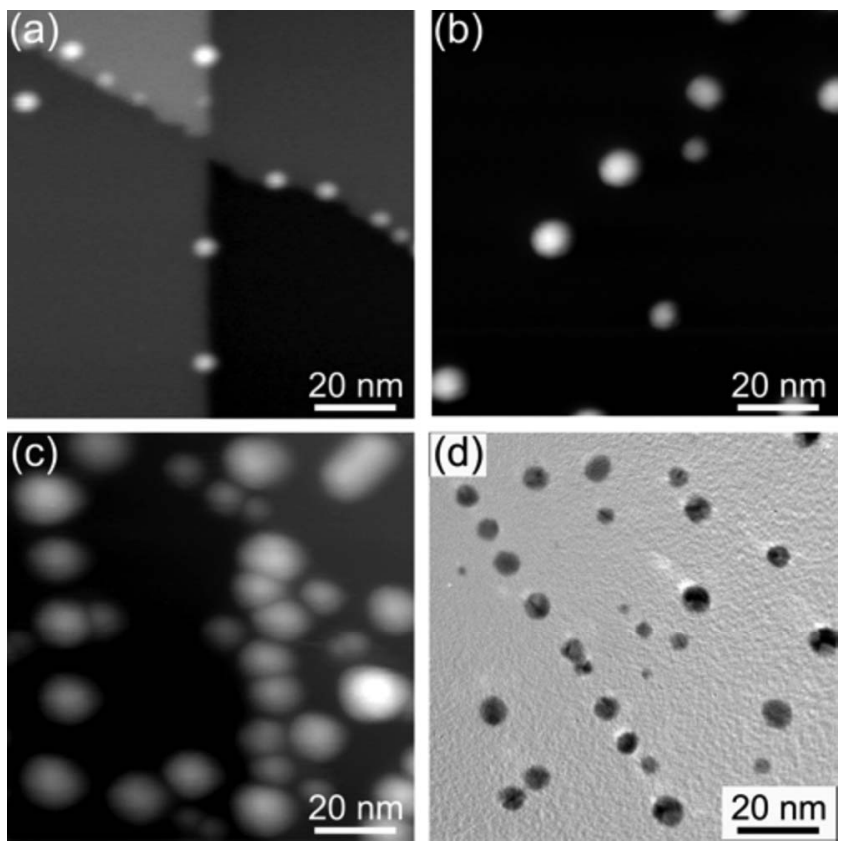

FIG. 1. Gold on $\mathrm{KBr}(001)$ measured by NC-AFM: (a) 0.0032 $\operatorname{ML}(\Delta f=-8.6 \mathrm{~Hz}),($ b) $0.064 \mathrm{ML}(\Delta f=-4.4 \mathrm{~Hz}),(\mathrm{c}) 0.64 \mathrm{ML}$ $(\Delta f=-2.9 \mathrm{~Hz})$, and (d) $0.64 \mathrm{ML}$, measured by TEM $(200 \mathrm{kV})$.

dence that the gold nanoparticles located at steps reside on the top side of the steps. This surprising result supports recent NC-AFM observations ${ }^{13,31}$ and earlier indirect evidence from double metal decoration TEM experiments. ${ }^{37}$ Adsorption at the lower side of the step would normally be expected owing to the increased coordination in comparison with the upper terrace. Szymonski and co-workers ${ }^{31}$ have proposed that $\mathrm{F}$ centers, i.e., electrons trapped in a halogen vacancies may accumulate along the steps and provide an additional binding energy. This is supported by calculations which have shown that $\mathrm{F}$ centers at steps provide significantly larger adhesion energies in comparison with regular step sites or terrace sites. ${ }^{38}$

In the case of the earlier studies, charge-induced desorption was used to intentionally pattern the surfaces with nanometer-scale pits and it has been suggested that residual surface defects such as F centers may remain at steps following the surface treatment. However, it is clear from Fig. 1(a) that the presence of gold particles on the upper side of $\mathrm{KBr}$ steps occurs on nonpatterned surfaces as well. It is likely that F centers are induced during the metal deposition, as it has been recently been shown that small amounts of charge emitted during evaporation can result in the generation of surface defects which act as nucleation centers. ${ }^{32,34}$ Some such defects may also result from the mechanical cleavage of the $\mathrm{KBr}(001)$ surface. ${ }^{39}$

Ex situ TEM measurements were also performed on one of the room-temperature-prepared gold nanoparticle samples [0.64 ML Au, Fig. 1(d)]. Owing to a lack of AFM-specific broadening effects, the TEM measurements more accurately reflect the lateral dimensions of the nanoparticles. Nevertheless, the TEM images do not provide height information. Moreover, TEM does not directly show the structure of the substrate on which the particles reside; only an outline of the 


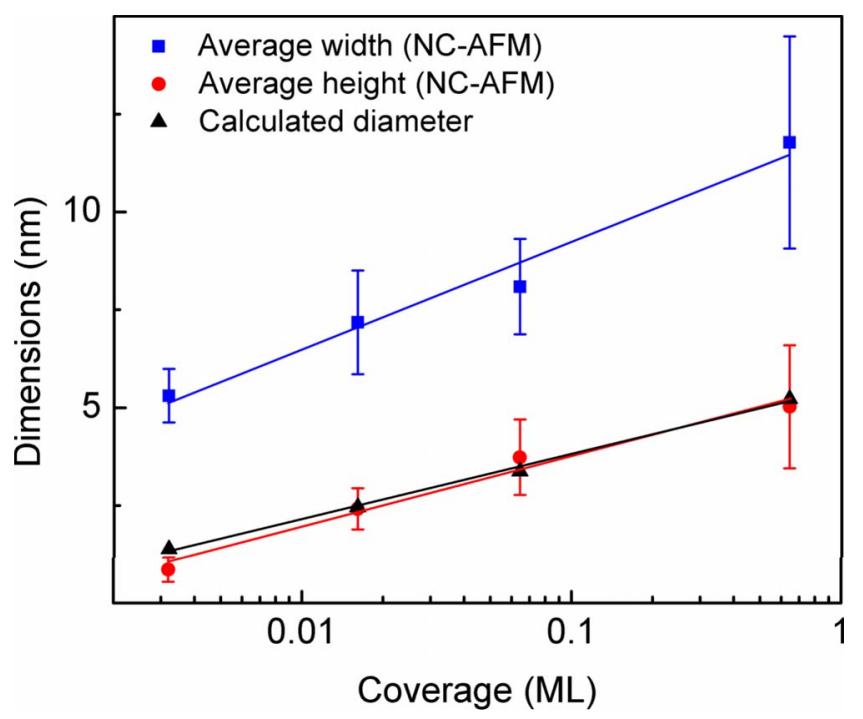

FIG. 2. (Color online) Dependence of the average nanoparticle dimensions (width and height) measured by NC-AFM. The nanoparticle diameters were also calculated based on the gold coverage, assuming a spherical nanoparticle morphology and a sticking coefficient of 1 .

$\mathrm{KBr}$ step structure can be inferred based on the preferred nucleation of the nanoparticles at step sites.

The average nanoparticle width (full width half maximum) and height at four gold coverages spanning from 0.0032 to $0.64 \mathrm{ML}$, measured by NC-AFM on terrace sites, are shown in Fig. 2. The heights and widths roughly follow an exponential dependence on coverage, with the average heights range from 0.9 to $5.0 \mathrm{~nm}$ and the average widths range from 5.3 to $11.8 \mathrm{~nm}$. The measured nanoparticle heights are expected to closely approximate the true heights. Small deviations may arise due to differences between the $\mathrm{KBr}$ and gold workfunctions ${ }^{40}$ and due to charging of the gold clusters, ${ }^{28,30,32}$ which would result in differing electrostatic forces over the gold and $\mathrm{KBr}$ regions. In order to reduce the overall electrostatic forces, a bias voltage corresponding to the minimum of a frequency shift versus voltage plot was applied to the sample. Provided the applied voltage is close to the mean contact potential of the sample, the correct heights will be measured. ${ }^{40}$ The lateral dimensions determined by NC-AFM, as in any AFM experiment on nonflat systems, are subject to tip convolution effects. The nanoparticle system is especially sensitive to such broadening since the dimensions of the nanoparticles are comparable with those of the tip apex. Moreover, the obtained widths are subject to greater variability than the heights as a result of the differing amounts of the tip broadening associated with the different tips used over the course of the experiments.

A comparison of the NC-AFM and TEM results at 0.64 ML coverage provides the opportunity to relate the height information obtained by NC-AFM with the lateral dimensions determined by TEM, making it possible to piece together the overall three-dimensional shape of the particles. The average height of $5.0 \mathrm{~nm}$ measured by NC-AFM very closely matches the average particle diameter of $5.1 \mathrm{~nm}$ measured by TEM. The size distributions are each represented by standard deviations of $1.6 \mathrm{~nm}$. The round appearance of the particles in both NC-AFM and TEM images, as well as the close correspondence between the measured heights and widths strongly suggests a nearly spherical particle shape. This is consistent with earlier high-resolution TEM observations which do not provide direct information about the three-dimensional proportions but reveal the crystal structure, which is predominantly a multiply twinned structure for metal nanoparticles on alkali halide surfaces at room temperature. ${ }^{41}$

MTP structures are widespread among nanoparticles composed of metals with face-centered-cubic lattices. These particles consist of slightly distorted tetrahedral subunits joined by twin boundaries. The energy of the creation of twin boundaries and the strain required for accommodating the new structure ${ }^{42}$ is low enough such that the total free energy can be reduced by taking on a nearly spherical shape bounded exclusively by low energy (111) planes. ${ }^{43}$ Several types are known to occur on alkali halides, including a pentagonal decahedron composed of five distorted twins and an icosahedron composed of 20 distorted twins. ${ }^{15}$ The presence of MTPs implies a very weak interaction with the substrate since particles with similar structures can be formed in inert atmospheres, such as argon gas. ${ }^{44}$ The fact that the observed particles exhibited highly similar heights and widths further supports the notion that the $\mathrm{KBr}$ substrate has a minimal influence on the overall three-dimensional particle shape and that the particles do not deviate significantly from their idealized symmetric crystal structure. Such behavior is also consistent with $a b$ initio theoretical studies of the interactions between noble metals and alkali halides which show that the interactions are primarily electrostatic and weak. ${ }^{45,46}$ The MTPs are probably slightly tilted on the substrate with one of the (111) facets lying along the plane of the substrate. ${ }^{43}$

Further evidence of a roughly spherical particle shape is provided by considering the particle dimensions expected based on the gold coverage. If a spherical particle shape and a sticking coefficient of 1 are assumed, calculated average nanoparticle diameters which very closely follow the measured average nanoparticle heights are obtained (see Fig. 2). The calculated value at $0.64 \mathrm{ML}$ also coincides with the TEM diameter measurement taken at this coverage. The selfconsistency of the calculations lends support to the spherical shape hypothesis for all of the measured coverages and strongly hints that the sticking coefficient at room temperature of gold on $\mathrm{KBr}$ is nearly unity. A sticking coefficient of about 1 has previously been noted at room temperature for the highly similar gold on $\mathrm{NaCl}(001)$ system. ${ }^{47}$ It should also be noted that the obtained nanoparticles have average diameters in the range which is of interest for gold catalysis, i.e., $1-5 \mathrm{~nm}^{3,4}$

The nearly spherical particle shape also makes it possible to estimate the radius of the tips used during the NC-AFM measurements. Treating the nanoparticles and the tip apex each as spheres, the tip radius $R_{T}$ can be estimated using ${ }^{48}$

$$
R_{T}=\frac{\left(W^{2}+4 h^{2}\right)}{8 h}-R_{p}
$$

where $W$ is the measured width at a given height $h$ from the top of the particle and $R_{p}$ is the true radius of the particle. 

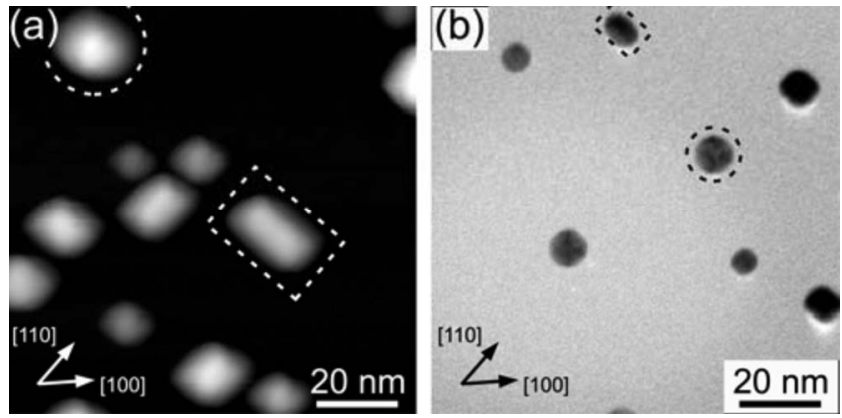

FIG. 3. Gold (0.64 ML) on $\mathrm{KBr}(001)$ prepared at elevated substrate temperatures: (a) NC-AFM $(\Delta f=-2.0 \mathrm{~Hz})$, sample prepared at $150{ }^{\circ} \mathrm{C}$, and (b) TEM $(200 \mathrm{kV})$, sample prepared at $250{ }^{\circ} \mathrm{C}$. Examples of MTPs and EPs are indicated by circles and rectangles, respectively.

This equation only applies in cases where the spherical apex, and not the side wall, is interacting with the particle. At the $0.64 \mathrm{ML}$ coverage, taking half the diameter measured by TEM as the true particle radius provides an estimated tip radius of $5.6 \mathrm{~nm}$. Similarly, a tip radius of $5.4 \pm 1.0 \mathrm{~nm}$ was determined for each of the measured coverages by considering the measured nanoparticle height or the calculated nanoparticle diameter as the true particle diameter. These relatively small tip radii compare favorably with the typical tip radius of under $7 \mathrm{~nm}$ and the guaranteed tip radius of under $10 \mathrm{~nm}$, specified by the manufacturer. It is likely that the reproducibly small radius is also partially due to the adopted procedure of rejecting tips exhibiting large tip-broadening effects.

Samples prepared at higher temperatures (150 and $250{ }^{\circ} \mathrm{C}$ ) exhibit particles of different shape (Fig. 3). Earlier TEM measurements have shown that gold nanoparticles on $\mathrm{KBr}$ and on $\mathrm{NaCl}$ come in two main forms: MTPs and EPs. ${ }^{41,43}$ As described above, MTPs are round particles which are composed of slightly distorted tetrahedral twins. The EPs are single crystals which are oriented along the $\langle 110\rangle$ directions in the case of growth on $\mathrm{KBr}(001)$. NCAFM measurements of such oriented rectangular EPs can be seen in Fig. 3(a). Round particles can be identified as MTPs. Not all particles, however, can be easily classified. Moreover, composite particles are also likely to be present on the surface. Our NC-AFM measurements confirmed the general trend that as the substrate temperature is increased during the evaporation, the proportion of EPs increases. ${ }^{41}$ The presence of EPs and MTPs was also verified by TEM [Fig. 3(b)]. With the exception of constant height mode measurements, ${ }^{29}$ previous NC-AFM measurements on similar systems have not revealed particle shapes other than domelike structures. These measurements demonstrate that the basic geometric shape of nanometer-scale particles can be resolved by NCAFM, provided the AFM tip is sufficiently sharp, in the constant frequency shift mode. The alignment of the particle facets in turn provides evidence for a crystalline particle structure and an epitaxial relationship between the gold and $\mathrm{KBr}$, which is confirmed by TEM observation.

\section{B. Tantalum on $\mathrm{KBr}(001)$}

Although tantalum is less commonly studied than gold, the growth of thin tantalum films has been studied exten-
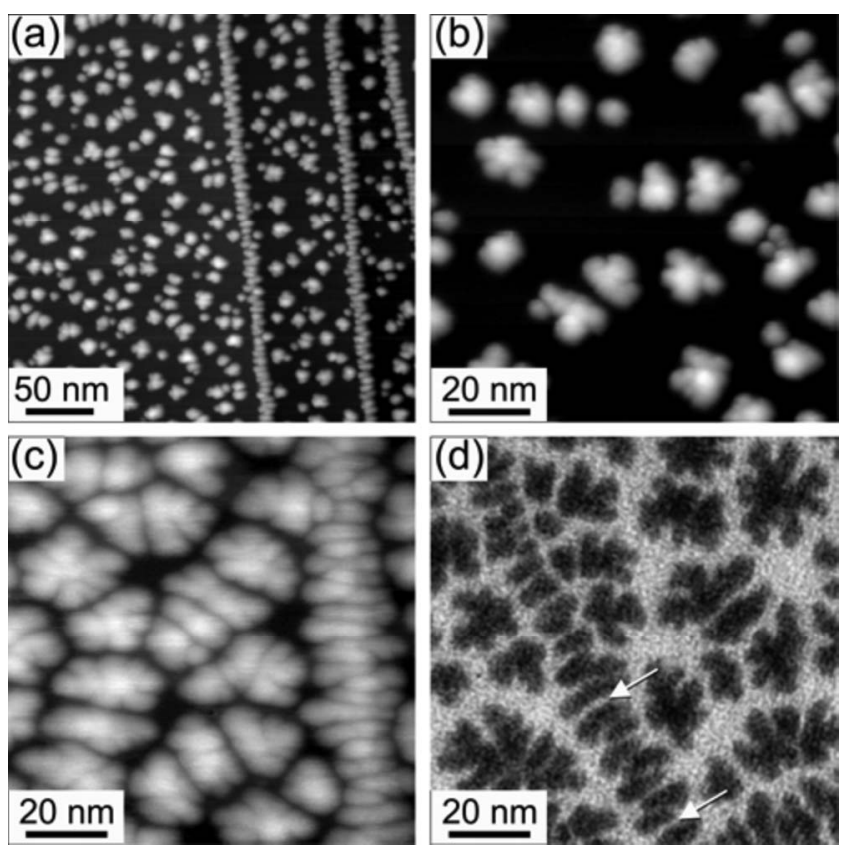

FIG. 4. Tantalum on $\mathrm{KBr}(001)$ at a coverage of $0.60 \mathrm{ML}$ measured by NC-AFM: (a) $(\Delta f=-4.0 \mathrm{~Hz})$ and (b) $\Delta f=-4.5 \mathrm{~Hz}$. A coverage of $1.8 \mathrm{ML}$ measured by (c) NC-AFM $(\Delta f=-3.9 \mathrm{~Hz})$ and (d) TEM $(80 \mathrm{kV})$. The arrows point to gaps between nanoparticles nucleated at a step.

sively for microelectronics applications. ${ }^{49}$ Tantalum was also selected because its bulk lattice constant is $0.33 \mathrm{~nm}$, which is precisely half the conventional lattice constant of $\mathrm{KBr}$, though epitaxial growth was not found.

Two tantalum coverages were considered, 0.60 and 1.8 ML. As seen in Fig. 4, this system follows the island growth mode; however, the island shapes show branching. Such structures are normally referred to as dendritic if the branches are symmetrically arranged and fractal if the structures are randomly ramified. ${ }^{50}$ Based on these definitions, the tantalum islands are fractal. This type of growth is common under conditions where the substrate temperature is less than about $15 \%$ of the melting temperature of the metal. ${ }^{51}$ For the case of tantalum, the substrate temperature of $20^{\circ} \mathrm{C}$ represents $8.9 \%$ of the melting temperature. Under these conditions, the diffusion of the tantalum atoms along the island edges is slow. As a result, an atom joining the island only samples a short length of the island perimeter before settling at a local energy minimum. The branching occurs because any protruding parts of the island block other parts, making them less accessible. Atoms arriving at random are then more likely to attach to the protruding structures than to find their way into the interstices. Moreover, the atom cannot diffuse far enough to move into and fill the interstices. The idealized case, where there is no edge diffusion at all, is referred to as diffusion-limited aggregation. The atom sticks to the island wherever it lands, resulting in one atom wide branching structures. In real growth, the branch width can be considerably wider, depending on the amount of edge diffusion. ${ }^{52}$

To the best of our knowledge, such fractal islands have not been previously observed in metal on insulator systems 
nor on a substrate with a square lattice. On metal substrates, fractal islands have only been obtained on lattices with triangular symmetry, whereas square lattices lead to compact islands. ${ }^{53}$ It is thought that this is a consequence of the incoming atoms relaxing into a local minimum following island impingement, with the incoming atom residing next to at least two atoms in the island. On a triangular lattice, this is compatible with branch formation; however, on a square lattice it is not. Interestingly, in the present case, fractal growth is manifested on a square lattice. It is expected that this is a result of the relatively weak interaction between the tantalum and the substrate, as evidenced by the island growth mode and the lack of preferential growth directions, which limits the influence of the square substrate lattice on the island structure.

The fractal dimension $D^{\prime}$ of the island perimeter can be quantified through the following relationship between the perimeter $P$ and area $A$,

$$
P=\mu A^{D^{\prime} / 2},
$$

where $\mu$ is a proportionality constant. ${ }^{54}$ The fractal dimension $D^{\prime}$ of the perimeter can vary between 1 and 2 . A larger value of $D^{\prime}$ reflects a more detailed structure of the island border. The fractal dimension was evaluated using the free WSXM software. ${ }^{55}$ Only nanoparticles on terrace sites were considered and particles which were cut off at the edges were excluded. Multiple thresholding values were used, and the obtained fractal dimensions averaged, for each image in order to eliminate qualitative bias in the determination of the island border. Moreover, TEM measurements [see, e.g., Fig. 4(d)] were favored over AFM measurements since the former does not suffer from broadening effects which could influence the measured island shape. At the $1.8 \mathrm{ML}$ coverage, $D^{\prime}$ was determined to be $1.53 \pm 0.08$. For comparison, the fractal dimension of gold particles at $0.64 \mathrm{ML}$ coverage was significantly smaller at $1.19 \pm 0.05$, owing to the simpler shape of the gold structures. It was also possible to extract a similar fractal dimension for the tantalum island perimeter based on the AFM data, $1.45 \pm 0.09$. The slightly lower $D^{\prime}$ relative to that measured from the TEM data reflects a loss of detail in the measured nanoparticle shape as a result of tip broadening.

The average height of the tantalum nanoparticles, on terrace sites, for a coverage of $0.60 \mathrm{ML}$ was $2.6 \mathrm{~nm}$ (with a standard deviation of $0.3 \mathrm{~nm}$ ), which is considerably smaller than the average height of $5.0 \mathrm{~nm}$ for gold nanoparticles at a similar coverage of $0.64 \mathrm{ML}$. This indicates that tantalum interacts more strongly with $\mathrm{KBr}$ than gold. In a theoretical study of the adsorption of three noble metals on $\mathrm{NaCl}$, it was shown by $a b$ initio methods that the interaction is mainly electrostatic and weak. ${ }^{45}$ This is largely because of the full valence $d$ orbitals present in the noble metals. It was suggested that metals which do not have completely filled valence $d$ orbitals would be able to bond with the substrate via mixing between the surface $p$ band and the metal $d$ orbitals. Furthermore, the absence of a closed valence orbital would lead to a reduction in the Pauli repulsion between the metal atom and the surface ions, and allow for greater orbital overlap. Since tantalum has only three electrons in its $5 d$ orbital,
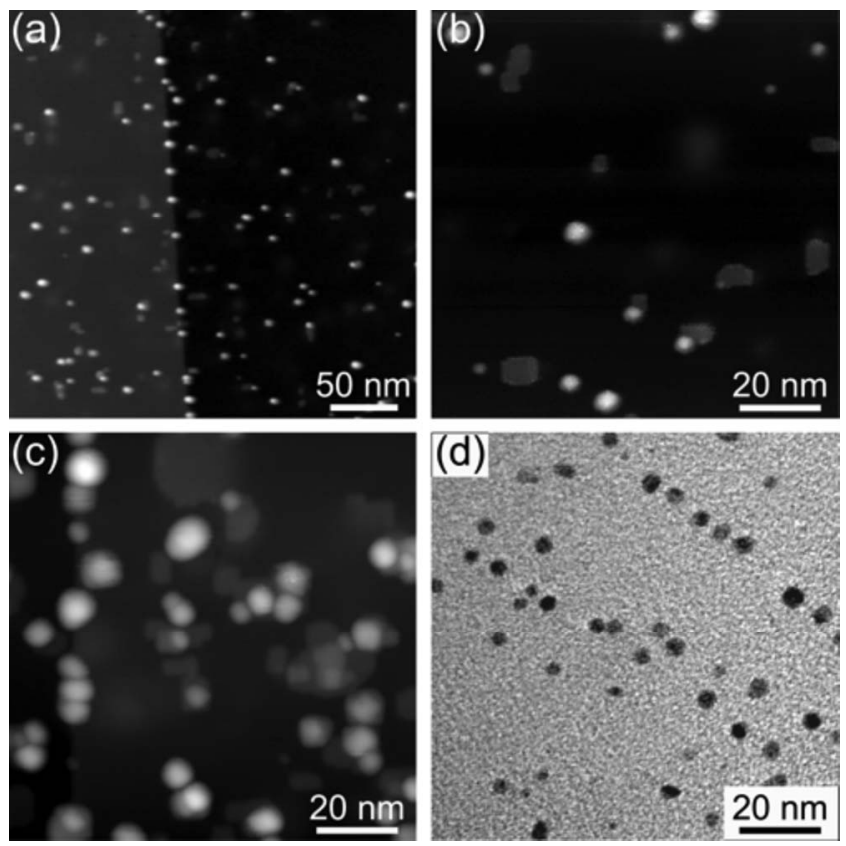

FIG. 5. NC-AFM images of palladium on $\mathrm{KBr}(001)$ at a coverage of $0.15 \mathrm{ML}$ measured by NC-AFM: (a) $\Delta f=-3.6 \mathrm{~Hz}$ and (b) $\Delta f=-4.0 \mathrm{~Hz}$. A coverage of $0.74 \mathrm{ML}$ measured by (c) NC-AFM $(\Delta f=-4.3 \mathrm{~Hz})$ and $(\mathrm{d})$ TEM $(200 \mathrm{kV})$.

it is likely that there is a chemical component to its bonding with the substrate, leading to an increased overall adsorbatesubstrate interaction. Nevertheless, the interaction is still insufficiently strong to induce epitaxial growth.

As seen in Fig. 4, the tantalum interacts particularly strongly with the $\mathrm{KBr}$ steps, leading to extensive step decoration. Unlike in the case of gold on $\mathrm{KBr}$ where the nanoparticles nucleate predominantly on the upper terrace of steps, the tantalum nanoparticles often straddle the steps or have a staggered positioning, providing evidence for nucleation at both the top and bottom sides. The resulting linear arrays of nanoparticles appear to form wirelike structures; however, careful inspection of the TEM measurements [Fig. 4(d)] shows that there are in fact gaps smaller than about 1 $\mathrm{nm}$ between some of the nanoparticles.

\section{Palladium on $\mathrm{KBr}(001)$}

Palladium is a particularly interesting metal for several reasons: insulator-supported palladium nanoparticles find prevalent use as catalysts, ${ }^{5}$ palladium can be used to store hundreds of times its volume in hydrogen for potential applications in hydrogen storage, ${ }^{56}$ palladium has been shown to have a tunable work function, depending on hydrogen exposure, and palladium has been used to produce ohmic contacts with carbon nanotubes, making it attractive for molecular electronics applications. ${ }^{57}$ Figure 5 shows the growth of palladium on the $\mathrm{KBr}(001)$ surface at two coverages, 0.15 and 0.74 ML. Much like with gold, the palladium nanoparticles form on terrace sites and on the upper side of atomic steps. In addition to the palladium nanoparticles, there are two other notable features: flat islands with $\langle 100\rangle$ edges and gradually protruding or hazy-looking areas. TEM measure- 

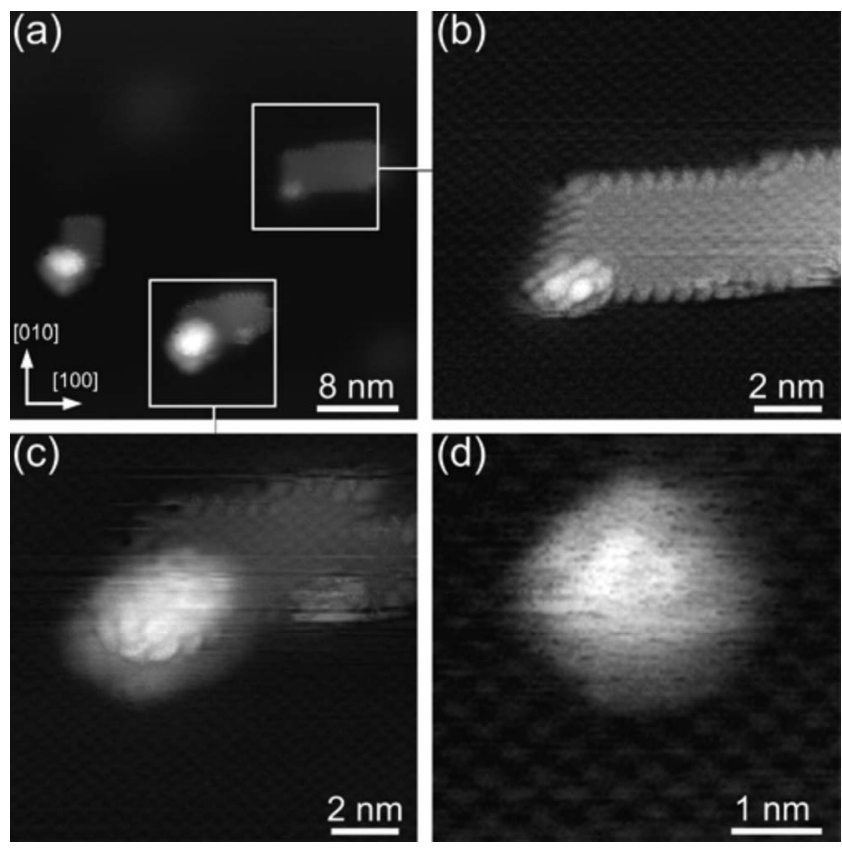

FIG. 6. High-resolution NC-AFM images showing palladium nanoparticles and flat $\langle 100\rangle$ faceted $\mathrm{KBr}$ islands: (a) $\Delta f=-2.4 \mathrm{~Hz}$, (b) $\Delta f=-2.9 \mathrm{~Hz}$, (c) $\Delta f=-2.0 \mathrm{~Hz}$, and (d) $\Delta f=-9.0 \mathrm{~Hz}$.

ments [Fig. 5(d)] provide no indication of these latter structures, revealing only the palladium nanoparticles.

High-resolution NC-AFM measurements of the flat islands [Figs. 6(a)-6(c)] show that they have the same lattice constant as the $\mathrm{KBr}$ substrate and the expected height of a $\mathrm{KBr}$ layer. Therefore, it is reasonable to conclude that the square-edged flat islands are in fact $\mathrm{KBr}$ islands with $\langle 100\rangle$ edges. The gradually protruding distortions in the $\mathrm{KBr}$ surface have measured heights which range from 0.1 to $0.3 \mathrm{~nm}$. These heights are similar to but smaller than the $\mathrm{KBr}$ step height of $0.33 \mathrm{~nm}$. We hypothesize that the protrusions may be caused by palladium which interdiffuses under the $\mathrm{KBr}$ surface. Moreover, this may point to a mechanism for the $\mathrm{KBr}$ island formation whereby some palladium atoms become embedded beneath the $\mathrm{KBr}$ surface, potentially causing a distortion of the lattice and the exchange of potassium and bromine ions to the surface. The diffusion of the palladium under the surface may be facilitated by the presence of pitlike $\mathrm{KBr}$ defect sites which are known to be produced by residual charged particles during deposition and to nucleate nanoparticle growth. ${ }^{32,34}$ Subsurface growth has previously been observed in certain metal on metal systems, although the mechanisms are only partially understood and appear to vary between systems. ${ }^{58,59}$ Such surface modifications could potentially be employed to purposely engineer the surface structure of the substrate for nanopatterning applications, much as nanometer-scale pits have previously been used to template metal and molecule growth on alkali halide surfaces. ${ }^{13,31,34,27,60}$

The high-resolution images in Figs. 6(a)-6(c) also show some protruding structures on top of the $\mathrm{KBr}$ island which have atomic-scale features with the same spacing as the $\mathrm{KBr}$ lattice. In the case of Fig. 6(c), the height of the protrusion is about $1 \mathrm{~nm}$. The large height relative to a $\mathrm{KBr}$ step suggests

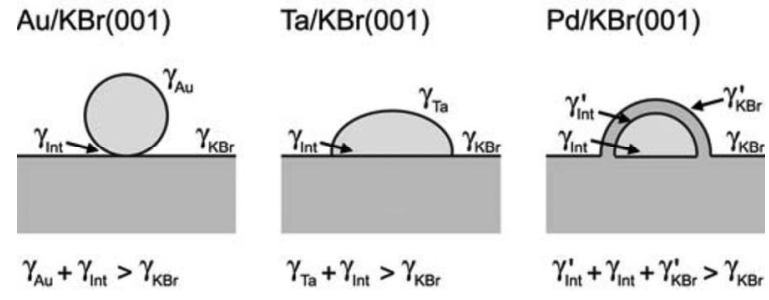

FIG. 7. Schematic of the island growth behavior observed in the $\mathrm{Au} / \mathrm{KBr}, \mathrm{Ta} / \mathrm{KBr}$, and $\mathrm{Pd} / \mathrm{KBr}$ systems along with the corresponding thermodynamic equilibrium inequalities relating the surface energies of the substrate, $\gamma_{\mathrm{KBr}}$, the surface energies of the metals, $\gamma_{\mathrm{Au}}$, $\gamma_{\mathrm{Ta}}, \gamma_{\mathrm{Pd}}$, and the interface energy $\gamma_{\text {Int }}$. In the case of the palladium, a film of substrate material encapsulates the metal, creating an additional surface and interface with corresponding energies denoted by $\gamma_{\mathrm{KBr}}^{\prime}$ and $\gamma_{\text {Int }}^{\prime}$

that the protrusion is in fact a palladium nanoparticle which is covered by a layer of $\mathrm{KBr}$. This interpretation is consistent with the notion that the palladium may embed itself under the $\mathrm{KBr}$ surface. Finally, some of the particles show alignment with the $\langle 110\rangle$ directions. One example is shown in Fig. 6(d). It is likely that this is an epitaxial palladium nanoparticle similar to those seen with gold on $\mathrm{KBr}(001)$. It is unclear whether all of the palladium particles are covered with $\mathrm{KBr}$. It is plausible that some are covered and some are not since the growth is a nonequilibrium process and the $\mathrm{KBr}$ is highly stable at room temperature.

\section{Surface and interface energies}

The types of growth observed in the three metal on $\mathrm{KBr}$ systems are summarized in Fig. 7. Although the growth behavior is partially influenced by kinetics, the main features can be explained by equilibrium thermodynamics considerations in terms of the surface and interface energies, i.e., the free energy per unit area required for creating a piece of surface or interface. In a system which follows the threedimensional island growth mode, also referred to as VolmerWeber growth, the surface energy of the deposited material $\gamma_{\text {dep }}$ plus the interface energy $\gamma_{\text {int }}$ is larger than the substrate surface energy $\gamma_{s u b}$,

$$
\gamma_{d e p}+\gamma_{\text {int }}>\gamma_{\text {sub }}
$$

The gold on KBr system, with its nearly spherical MTP gold nanoparticle structure, clearly follows this growth mode, which is to be expected given the very large surface energy of gold $\left(1.3 \mathrm{~J} / \mathrm{m}^{2}\right)$ (Ref. 61) compared with the surface energy of $\mathrm{KBr}(001)\left(0.15 \mathrm{~J} / \mathrm{m}^{2}\right) .{ }^{62} \mathrm{In}$ addition, the nanoparticle surface area is significantly larger than the interfacial surface area, which provides evidence that the energy cost of creating a gold $/ \mathrm{KBr}$ interface is greater than the energy required for forming a gold surface. That is, $\gamma_{\text {int }}>\gamma_{d e p}$ or $\gamma_{\text {int }}$ $>1.3 \mathrm{~J} / \mathrm{m}^{2}$ for gold on $\mathrm{KBr}(001)$.

Tantalum has a surface energy of $2.5 \mathrm{~J} / \mathrm{m}^{2},{ }^{61}$ significantly larger than the surface energy of $\mathrm{KBr}\left(0.15 \mathrm{~J} / \mathrm{m}^{2}\right)$, therefore, at least according to equilibrium considerations [i.e., inequality (3)], island growth is to be expected. The tantalum had a flatter, more spread out island morphology in 
comparison with gold, which suggests an increased interaction of tantalum with the $\mathrm{KBr}$ surface and a reduced energy cost for creating interfacial area. More precisely, the flatter island shape indicates that inequality (3) is satisfied such that $\gamma_{\text {dep }}+\gamma_{\text {int }}$ is more comparable to $\gamma_{s u b}$ (the system is closer to the conditions for layer-by-layer growth) or $\gamma_{\mathrm{Ta}}+\gamma_{\mathrm{Int}(\mathrm{Ta})}$ $<\gamma_{\mathrm{Au}}+\gamma_{\text {Int }(\mathrm{Au})}$. Inserting the known tantalum $\left(2.5 \mathrm{~J} / \mathrm{m}^{2}\right)$ and gold $\left(1.3 \mathrm{~J} / \mathrm{m}^{2}\right)$ surface energies leads to the condition that the interfacial energy of tantalum is at least $1.2 \mathrm{~J} / \mathrm{m}^{2}$ smaller than the interfacial energy of gold. It must be kept in mind that this is approximate, especially since it is clear that the tantalum structures are not in equilibrium, as evidenced by their fractal shape.

The case of palladium on $\mathrm{KBr}(001)$ is peculiar in that it appears that the overall system energy is reduced by coating the deposited material with a layer of the substrate material. This situation is described by the following surface energy relationship ${ }^{58}$

$$
\gamma_{\text {int }}^{\prime}+\gamma_{\text {int }}+\gamma_{\text {sub }}^{\prime}>\gamma_{\text {sub }},
$$

where $\gamma_{\text {sub }}^{\prime}$ and $\gamma_{\text {int }}^{\prime}$ are the substrate and interface energies associated with the new surface and interface introduced by the substrate material coating (see Fig. 7). These are expected to be similar to the conventional surface and interface energies but may deviate slightly due to structural differences. Since substrate-coated islands are favored over bare islands, by comparing condition (3) for bare particles and condition (4) for covered islands, we obtain the inequality

$$
\gamma_{\text {int }}^{\prime}+\gamma_{s u b}^{\prime}<\gamma_{d e p}
$$

Inequality (5) provides the conditions under which the system energy can be reduced by coating the deposited material with a layer of the substrate. Inserting the surface energy of palladium $\left(1.7 \mathrm{~J} / \mathrm{m}^{2}\right)$ and assuming that the surface energy of the thin $\mathrm{KBr}$ coating is similar to a regular $\mathrm{KBr}$ surface $\left(0.15 \mathrm{~J} / \mathrm{m}^{2}\right)$, we obtain the requirement that the interface energy is under $1.55 \mathrm{~J} / \mathrm{m}^{2}$.

Given the large surface energy of most metals and the small surface energy of the $\mathrm{KBr}$ and that of other alkali halides, island growth is expected for a majority of metal on alkali halide combinations [inequality (3)]. It is likely that the condition for buried islands [inequality (5)] is satisfied in many cases, provided that the associated interface energy is sufficiently small.

\section{E. Imaging considerations}

While atomic resolution NC-AFM measurements were readily achieved on the $\mathrm{KBr}$ substrate, on $\mathrm{KBr}$ islands, and on palladium particles covered by $\mathrm{KBr}$, it was not possible to resolve the atomic structure of the gold or tantalum nanoparticles. Such systems are particularly challenging to image for several reasons. First, previous reports of NC-AFM measurements on atomically flat metal surfaces show extremely small atomic corrugations of 10-20 p.m. ${ }^{23,24}$ These height variations are minuscule in comparison with the $1-5 \mathrm{~nm}$ height of the nanoparticles, making it exceedingly difficult to achieve optimal feedback conditions which are stable and responsive to both length scales.
Tip instabilities have also been observed due to sudden tip changes, which have occasionally been associated with the disappearance of a nanoparticle from the surface. In the latter case, it is most likely that the particle is transferred to the AFM tip. This is not surprising in the case of gold, where the nearly spherical MTPs are weakly coupled to the surface. In some instances, the tip changes seen during NC-AFM imaging of metal nanoparticles have caused significant changes in the tip-sample interaction, making subsequent imaging highly unstable. This suggests that either the tip apex interacts in an unstable manner with the surface or that the adsorbates on the tip apex are not firmly fixed. According to recent simulations, a tip with gold atoms at the apex is highly prone to instabilities due to atomic jumps between the surface and the tip. ${ }^{38}$ Moreover, the calculations showed that $\mathrm{K}^{+}$-terminated tips interact too weakly with gold nanoparticles to achieve atomic resolution and $\mathrm{Br}^{-}$-terminated tips, like the gold tips, are unstable at the small tip-sample separations required for high-resolution imaging.

Tip convolution also plays a role in preventing atomic resolution in NC-AFM measurements of nanoparticles. As the tip makes its way over the contour of a nanoparticle, different faces of the tip apex interact with the particle. This is in contrast with the situation on flat surfaces where highresolution information is provided by the sustained shortrange interaction between a single sharp asperity at the tip apex and the sample. Moreover, when the tip is over the side portion of a nanoparticle, the geometry is such that some parts of the tip interact with the nanoparticle while other parts interact with the substrate, leading to a mixture of the two signals. Over a small region directly on the top of the nanoparticle, tip convolution is expected to play a lesser role.

Finally, as shown by Barth and Henry, ${ }^{28}$ there is a significant measurable contact potential difference between the gold and the $\mathrm{KBr}$. Therefore, there is an electrostatic component in the measurements which may dominate over the short-range interactions responsible for atomic resolution. Attempts have been made in our experiments and in others ${ }^{30}$ to employ Kelvin probe feedback in order to dynamically minimize the electrostatic interactions; however this has not yet provided any clear improvements in resolution.

Overall, for measuring the structure of metal nanoparticles on insulating substrates, NC-AFM offers the advantages that the measurements are performed in situ, provide direct topographic information about the nanoparticle dimensions and locations, allow for accurate nanoparticle height determination, and provide high (often atomic) resolution information about the substrate structure. The main disadvantage of the NC-AFM approach is the tip broadening of the lateral particle dimensions and the lack of information about the atomic arrangement within the nanoparticles. On the other hand, TEM provides accurate lateral dimensions but no height information. High-resolution TEM can be used to offer details about metal nanoparticle crystal structure; however, it should be noted that in the size range considered here, 1-5 nm, structural instabilities due to electron-beaminduced sintering can become problematic. ${ }^{63}$ Moreover, there are concerns about sample integrity for the TEM measurements because they are normally performed ex situ on carbon-coated replicas which may be affected by contamina- 
tion or may slightly deform and thus not fully accurately reflect the original nanoparticle shape or position. Finally, TEM only provides indirect information about the substrate structure. The presence of extended surface steps can generally be inferred via the presence of nanoparticle rows; however, small islands or defects generally cannot be distinguished. This was especially evident in the case of palladium on $\mathrm{KBr}$ where NC-AFM measurements revealed considerable surface modification which was undetectable by TEM. Clearly, the two imaging techniques are highly complementary with each having its own set of advantages and limitations.

\section{SUMMARY AND CONCLUSIONS}

The early stages of the growth of gold, tantalum, and palladium on the $\mathrm{KBr}(001)$ surface were investigated under ultrahigh vacuum conditions by $\mathrm{NC}-\mathrm{AFM}$ and ex situ by TEM. Through comparison of the NC-AFM and TEM results, details about the three-dimensional particle morphology were obtained with the NC-AFM measurements offering reliable height information and the TEM measurements providing accurate lateral dimensions.

The three metal on insulator systems exhibited highly varied growth morphologies. At room temperature, the gold nanoparticles were shown to have a nearly spherical shape, which is consistent with the previously established multiply twinned crystal structure and a very weak interaction with the substrate. The diameters of the particles were in the 1-5 $\mathrm{nm}$ range, which coincides with the relevant size for applications such as catalysis. Because the gold particles were nearly spherical it was also possible to estimate the radius of the AFM tips employed during the measurements, which were in the range of $5.4 \pm 1.0 \mathrm{~nm}$. At higher temperatures, an increasing number of $\langle 110\rangle$-oriented epitaxial gold nanoparticles was observed by TEM; in addition, the rectangular shape of the nanoparticles was visualized by NC-AFM. Tantalum grew as ramified islands with a flat shape in comparison with the gold nanoparticles, indicating limited diffusion of tantalum along island edges and a stronger substrate interaction relative to the gold $/ \mathrm{KBr}$ system. Despite this, an overall weak substrate interaction prevails, as evidenced by the island growth mode and the absence of a preferential growth direction of the particle branches. This is expected to decouple the overlayer structure from the substrate, enabling the fractal growth which is not normally observed on a square lattice. The deposition of palladium yielded some unexpected behavior with the generation of $\mathrm{KBr}$ islands and gradual protrusions in the substrate accompanying the palladium nanoparticle growth. It is thought that these surface features are induced by the interdiffusion of palladium atoms beneath the $\mathrm{KBr}$ surface. Consideration of the involved surface energies yielded bounds on the metal/KBr interface energies and provided an outline of the conditions under which island and buried island growth is to be expected. Given the typical range of alkali halide and metal surface energies, most metal on alkali halide systems are expected to follow one of these two growth modes.

NC-AFM also gave direct information about the substrate which was inaccessible by TEM: gold and palladium nanoparticles were shown to nucleate at the top side of atomic steps while tantalum nanoparticles had a high affinity for the substrate steps and nucleated at both the top and bottom sides, forming linear chains of nanoparticles. Palladium growth on $\mathrm{KBr}$ (001) also led to the peculiar evolution of additional $\mathrm{KBr}$ islands on the substrate surface as well as the creation of gradual protrusions which were invisible to TEM observation. These measurements highlight the critical role of the metal-substrate interaction in determining the nanoparticle growth behavior and the importance of the NC-AFM approach in elucidating the influence of the substrate during nanoparticle growth on insulators.

\section{ACKNOWLEDGMENTS}

The authors thank H. Vali for recording the TEM images. The following funding agencies are gratefully acknowledged for support: NSERC, CIfAR, FQRNT, and CFI.

\footnotetext{
*Present address: Institut de Science et d'Ingénierie Supramoléculaires (ISIS)-CNRS 7006, Université de Strasbourg, 8 allée Gaspard Monge, 67000 Strasbourg, France.

${ }^{\dagger}$ Present address: Department of Physics, UC Berkeley, Berkeley, CA 94720-7300, USA.

${ }^{1}$ A. N. Shipway, E. Katz, and I. Willner, ChemPhysChem 1, 18 (2000).

${ }^{2}$ P. K. Jain, I. H. El-Sayed, and M. A. El-Sayed, Nanotoday 2, 18 (2007).

${ }^{3}$ M. Valden, X. Lai, and D. W. Goodman, Science 281, 1647 (1998).

${ }^{4}$ M. Haruta, Gold Bull. 37, 27 (2004).

${ }^{5}$ C. R. Henry, Surf. Sci. Rep. 31, 231 (1998).

${ }^{6}$ J. A. Venables, G. D. T. Spiller, and M. Hanbucken, Rep. Prog. Phys. 47, 399 (1984).

${ }^{7}$ C. L. Cheung, A. Kurtz, H. Park, and C. M. Lieber, J. Phys.
}

Chem. B 106, 2429 (2002).

${ }^{8}$ S. Helveg, C. Lopez-Cartes, J. Sehested, P. L. Hansen, B. S. Clausen, J. R. Rostrup-Nielsen, Frank Abild-Pedersen, and Jens K. Nørskov, Nature (London) 427, 426 (2004).

${ }^{9}$ K. A. Dick, K. Deppert, L. S. Karlsson, L. R. Wallenberg, L. Samuelson, and W. Seifert, Adv. Funct. Mater. 15, 1603 (2005).

${ }^{10}$ J. B. Hannon, S. Kodambaka, F. M. Ross, and R. M. Tromp, Nature (London) 440, 69 (2006).

${ }^{11}$ A. Bezryadin, C. Dekker, and G. Schmid, Appl. Phys. Lett. 71, 1273 (1997).

${ }^{12}$ T. Morita and S. Lindsay, J. Am. Chem. Soc. 129, 7262 (2007).

${ }^{13}$ J. M. Mativetsky, S. A. Burke, S. Fostner, and P. Grutter, Small 3, 818 (2007).

${ }^{14}$ G. A. Bassett, Philos. Mag. 3, 1042 (1958).

${ }^{15}$ S. Ogawa and S. Ino, J. Cryst. Growth 13-14, 48 (1972).

${ }^{16}$ M. Bammerlin, R. Lüthi, E. Meyer, A. Baratoff, J. Lü, M. Gug- 
gisberg, C. Loppacher, C. Gerber, and H.-J. Güntherodt, Appl. Phys. A: Mater. Sci. Process. 66, S293 (1998).

${ }^{17}$ R. Bennewitz, S. Schar, V. Barwich, O. Pfeiffer, E. Meyer, F. Krok, B. Such, J. Kolodzej, and M. Szymonski, Surf. Sci. 474, L197 (2001).

${ }^{18}$ R. Hoffmann, M. A. Lantz, H. J. Hug, P. J. A. van Schendel, P. Kappenberger, S. Martin, A. Baratoff, and H. -J. Güntherodt, Appl. Surf. Sci. 188, 238 (2002).

${ }^{19}$ Clemens Barth and Claude R. Henry, Phys. Rev. Lett. 91, 196102 (2003).

${ }^{20}$ F. J. Giessibl, Science 267, 68 (1995).

${ }^{21}$ S. Kitamura and M. Iwatsuki, Jpn. J. Appl. Phys., Part 2 34, L145 (1995)

${ }^{22}$ M. A. Lantz, H. J. Hug, R. Hoffmann, P. J. A. van Schendel, P. Kappenberger, S. Martin, A. Baratoff, and H.-J. Güntherodt, Science 291, 2580 (2001).

${ }^{23}$ S. Orisaka, T. Minobe, T. Uchihashi, Y. Sugawara, and S. Morita, Appl. Surf. Sci. 140, 243 (1999).

${ }^{24}$ Ch. Loppacher, M. Bammerlin, M. Guggisberg, S. Schär, R. Bennewitz, A. Baratoff, E. Meyer, and H. J. Güntherodt, Phys. Rev. B 62, 16944 (2000).

${ }^{25}$ T. Uchihashi, T. Ishida, M. Komiyama, M. Ashino, Y. Sugawara, W. Mizutani, K. Yokoyama, S. Morita, H. Tokumoto, and M. Ishikawa, Appl. Surf. Sci. 157, 244 (2000).

${ }^{26}$ S. A. Burke, J. M. Mativetsky, R. Hoffmann, and P. Grutter, Phys. Rev. Lett. 94, 096102 (2005).

${ }^{27}$ J. M. Mativetsky, S. A. Burke, S. Fostner, and P. Grutter, Nanotechnology 18, 105303 (2007).

${ }^{28}$ C. Barth and C. R. Henry, Nanotechnology 15, 1264 (2004).

${ }^{29}$ C. Barth, O. H. Pakarinen, A. S. Foster, and C. R. Henry, Nanotechnology 17, S128 (2006).

${ }^{30}$ C. Barth and C. R. Henry, Appl. Phys. Lett. 89, 252119 (2006).

${ }^{31}$ M. Goryl, F. Buatier de Mongeot, F. Krok, A. Vevecka-Priftaj, and M. Szymonski, Phys. Rev. B 76, 075423 (2007).

${ }^{32}$ J. M. Mativetsky, S. Fostner, S. A. Burke, and P. Grutter, Surf. Sci. 602, L21 (2008).

${ }^{33}$ O. H. Pakarinen, C. Barth, A. S. Foster, and C. R. Henry, J. Appl. Phys. 103, 054313 (2008).

${ }^{34}$ J. M. Mativetsky, Y. Miyahara, S. Fostner, S. A. Burke, and P. Grutter, Appl. Phys. Lett. 88, 233121 (2006).

${ }^{35}$ S. Morita, R. Wiesendanger, and E. Meyer, Noncontact Atomic Force Microscopy (Springer, Berlin, 2002).

${ }^{36}$ T. R. Albrecht, P. Grutter, D. Horne, and D. Rugar, J. Appl. Phys. 69, 668 (1991).

${ }^{37} \mathrm{H}$. Bethge, in Interfacial Aspects of Phase Transformations, Proceedings of the NATO Advanced Study Institute, Erice, Sicily, 29 August-9 September 1981, edited by B. Mutaftschiev (D. Reidel, Dordrecht, Holland, 1982), p. 669.

${ }^{38}$ O. H. Pakarinen, C. Barth, A. S. Foster, R. M. Nieminen, and C.
R. Henry, Phys. Rev. B 73, 235428 (2006).

${ }^{39}$ C. Barth and C. R. Henry, Nanotechnology 17, S155 (2006).

${ }^{40} \mathrm{~S}$. Sadewasser and M. C. Lux-Steiner, Phys. Rev. Lett. 91, 266101 (2003).

${ }^{41}$ K. Yamamoto, T. Iijima, T. Kunishi, K. Fuwa, and T. Osaka, J. Cryst. Growth 94, 629 (1989).

${ }^{42}$ C. L. Johnson, E. Snoeck, M. Ezcurdia, B. Rodriguez-Gonzalez, I. Pastoriza-Santos, L. M. Liz-Marzan, and Martin J. Hÿtch, Nature Mater. 7, 120 (2008).

${ }^{43}$ H. Sato and S. Shinozak, Surf. Sci. 22, 229 (1970).

${ }^{44}$ K. Kimoto and I. Nishida, J. Phys. Soc. Jpn. 22, 940 (1967).

${ }^{45}$ J. A. Mejias, Phys. Rev. B 53, 10281 (1996).

${ }^{46}$ M. H. Hakala, O. H. Pakarinen, and A. S. Foster, Phys. Rev. B 78, 045418 (2008).

${ }^{47}$ R. Conrad and M. Harsdorff, Thin Solid Films 192, 163 (1990).

${ }^{48}$ K. A. Ramirez-Aguilar and K. L. Rowlen, Langmuir 14, 2562 (1998).

${ }^{49}$ W. D. Westwood, N. Waterhouse, and P. S. Wilcox, Tantalum Thin Films (Academic, London, New York, 1975).

${ }^{50}$ H. Brune, C. Romainczyk, H. Roder, and K. Kern, Nature (London) 369, 469 (1994).

${ }^{51}$ T. Michely and J. Krug, Islands, Mounds, and Atoms: Patterns and Processes in Crystal Growth Far From Equilibrium (Springer, Berlin, New York, 2004).

${ }^{52}$ K. Oura, Surface Science: An Introduction (Springer, Berlin, 2003).

${ }^{53}$ Z. Y. Zhang and M. G. Lagally, Science 276, 377 (1997).

${ }^{54}$ R. F. Voss, R. B. Laibowitz, and E. I. Allessandrini, Phys. Rev. Lett. 49, 1441 (1982).

${ }^{55}$ I. Horcas, R. Fernandez, J. M. Gomez-Rodriguez, J. Colchero, J. Gomez-Herrero, and A. M. Baro, Rev. Sci. Instrum. 78, 013705 (2007).

${ }^{56}$ C. Sachs, A. Pundt, R. Kirchheim, M. Winter, M. T. Reetz, and D. Fritsch, Phys. Rev. B 64, 075408 (2001).

${ }^{57}$ A. Javey, J. Guo, Q. Wang, M. Lundstrom, and H. J. Dai, Nature (London) 424, 654 (2003).

${ }^{58}$ C. Nagl, E. Platzgummer, M. Schmid, P. Varga, S. Speller, and W. Heiland, Surf. Sci. 352-354, 540 (1996).

${ }^{59}$ P. W. Murray, S. Thorshaug, I. Stensgaard, F. Besenbacher, E. Lægsgaard, A. V. Ruban, K. W. Jacobsen, G. Kopidakis, and H. L. Skriver, Phys. Rev. B 55, 1380 (1997).

${ }^{60}$ L. Nony, E. Gnecco, A. Baratoff, A. Alkauskas, R. Bennewitz, O. Pfeiffer, S. Maier, A. Wetzel, E. Meyer, and Ch. Gerber, Nano Lett. 4, 2185 (2004).

${ }^{61}$ W. R. Tyson and W. A. Miller, Surf. Sci. 62, 267 (1977).

${ }^{62}$ F. van Zeggeren and G. C. Benson, J. Chem. Phys. 26, 1077 (1957).

${ }^{63}$ S. Iijima and T. Ichihashi, Phys. Rev. Lett. 56, 616 (1986). 